\title{
Fibrinogenolytic Proteases Isolated from the Snake Venom of Taiwan Habu: Serine Proteases with Kallikrein-like and Angiotensin-Degrading Activities ${ }^{1}$
}

\author{
Chin-Chun Hung* and Shyh-Horng Chiou*' $\dagger^{2}$ \\ *I nstitute of Biochemical Sciences, College of Science, National Taiwan University, Taipei, Tai wan; and \\ †I nstitute of Biological Chemistry, P.O. Box 23-106, Academia, Taipe, Taiwan
}

Received J anuary 16, 2001

Two venom proteases with fibrinogenolytic activity were isolated from the venom of Taiwan habu (Trimeresurus mucrosquamatus), one major crotalid snake species in Taiwan. The purified enzymes showed a strong $\beta$-fibrinogenolytic activity, cleaving $\beta$-chain of fibrinogen molecules specifically. They also showed strong kallikrein-like activity in vitro, releasing bradykinin from kininogen. The purified enzymes did not coagulate human plasma, yet decreasing fibrinogen levels in plasma and prolonging bleeding without formation of fibrin clots, indicating that both proteases have specificities different from thrombin and thrombin-like proteases of snake venom reported previously. They also exhibit amidase activity against $\mathbf{N}$-benzoyl-Pro-Phe-Arg-p-nitroanilide, which is a specific synthetic substrate for kallikrein-like proteases. Their stability at high temperatures was examined and found to be more stable when compared with ancrod and thrombin. Intravenous injection of either protease was shown to lower blood pressure in experimental rats. Most noteworthy is the observation that the proteases can cleave angiotensin I and release bradykinin from plasma kininogen in vitro, which is a strong vasodi lator and probably responsible for the in vivo hypotensive effect of these venom proteases. ○ 2001 Academic Press

Key Words: $\beta$-fibrinogenolytic activity; kallikreinlike proteases; bradykinin; angiotensin I; hypertension therapy.

Venoms from various snake species alter the haemostatic and blood coagulation systems of human victims

\footnotetext{
${ }^{1}$ The cDNA sequences encoding similar venom serine proteases to Tm-VIG and Tm-IIG isolated from Taiwan habu (Trimeresurus mucrosquamatus) have been deposited in the EMBO database under the Accession Nos. X83221 and X83225.

${ }^{2}$ To whom correspondence should be addressed. Fax: (886)-226530014. E-mail: shchiou@gate.sinica.edu.tw.
}

or experimental animals in a complex manner. Different venoms contain multiple components which behave as pro- or anticoagulants that directly (or indirectly) induce or inhibit fibrinogen and/or platel et aggregation and related complex biochemical processes, resulting in common clinical complications of blood clotting or uncontrolled hemorrhage by envenomation of snakebites (1-3). These apparently contrasting activities have been attributed to the presence of fibrinogenolytic or fibrinogen clotting enzymes in snake venoms (4-6). On the other hand, platelet-aggregating enzymes in venom generally lack fibrinogenolytic activity, but can directly aggregate platel ets in platel et-rich plasma (7). Current interest is directed to some fibrinolytic proteinases including metalloproteinases and thrombin-like enzymes because of their potential clinical application in the treatment of vascular thrombotic diseases (8).

It is well known that snake venoms contain complex mixtures of pharmacologically active peptides and proteins. Reptilian venoms particularly those obtained from the snake families of Crotalidae and Viperidae, are also shown to possess many different fibrinogenolytic proteases which may initiate or affect blood coagulation process associated with snakebites (6). Different groups reported disparate proteases from venoms of various crotalid snakes. They included crotalase, a thrombin-like enzyme isolated from the American-E astern diamondback rattlesnake (Crotalus adamanteus) (9), hemorrhagic toxins, anticoagulant proteases and kallikrein-like enzymes from the AmericanWestern diamondback rattlesnake Crotalus atrox (1012). We have previously evaluated the venom components from Crotalus atrox and found that all fractions isolated from the anion-exchange chromatography showed varying extents of specific proteolytic activity against $\alpha$ - and/or $\beta$-chains of fibrinogen molecules (13, 14). Concurrently, studies on the toxin components from Taiwan habu (Trimeresurus mucrosquamatus) 
(15-17), a major and abundant crotalid species in Taiwan, indicated several kinds of fibrinogenases present in this phylogenetically related species to those American rattlesnakes.

Concerning the pharmacological action of Formosan snake venoms on blood coagulation, it was reported early in 1921-1925 that the crude venoms of two crotalid snake species, Agkistrodon acutus and Trimeresurus gramineus, had a coagulant action on whole blood and plasma, while the venom of another species Trimeresurus mucrosquamatus of the same family showed an inhibitory action (1). The inhibitory action on blood coagulation was believed to be caused mostly by destruction of fibrinogen in the case of the venom of Trimeresurus mucrosquamatus. Therefore it is deemed imperative to isol ate venom enzymes which are responsible for these fibrinogen-degrading activities that destroy the precursor fibrinogen molecules with the result of excluding the formation of fibrin cl ot in the blood plasma. In this study we have made an effort in the search and characterization of these fibrinogenases from Taiwan habu, which show an unexpectedly strong kallikrein-like hypotensive activity upon experimental rats and may find their clinical applications in hypertension therapy.

\section{MATERIALS AND METHODS}

Crude venom and chemicals for protein isolation and purification. The lyophilized venom powder was obtained from the local snake farm, and the venom gland of Taiwan habu was donated from the National Institute of Preventive Medicine, Taipei, Taiwan. The synthetic substrates, human fibrinogen, angiotensin I, bradykinin, kallikrein, snake venoms of other species and various protease inhibitors were from Sigma Chemical Co. (St. Louis, MO). High molecular weight kininogen was obtained from Enzyme Research Laboratories Inc. (South Bend, IN).

Thermostability study of purified proteases. The thermostability for purified kallikrein-like fibrinogenases together with ancrod and human thrombin was determined by pre-incubating enzymes in $0.1 \mathrm{M}$ Tris, pH 8.0 buffer, at $25,35,45,55,65,75,85$, and $95^{\circ} \mathrm{C}$ for $30 \mathrm{~min}$. After preincubation, the enzyme was mixed with synthetic substrates and proteolytic activities then determined on a spectrophotometer by measuring absorbances at $A_{405 \mathrm{~nm}}$. The amidolytic activity towards various chromogenic substrates was measured with a UItrospec 4000 spectrophotometer (Amersham/Pharmacia) in a plastic cuvette with 1-cm path length. Assays were performed in $50 \mathrm{mM}$ Tris- $\mathrm{HCl}, \mathrm{pH} 8.0$ in a total volume of $750 \mu \mathrm{l}$ at $37^{\circ} \mathrm{C}$. The final concentrations of enzymes were $1.0 \mathrm{nM}$ for Tm-VIG and Tm-IIG, $0.02 \mathrm{U} / \mathrm{ml}$ for human thrombin (930 $\mathrm{NIH} \mathrm{U/mg} \mathrm{protein} \mathrm{based} \mathrm{on} \mathrm{Biuret} \mathrm{assays)} \mathrm{and} 0.04 \mathrm{U} / \mathrm{ml}$ for Ancrod (500 NIH U/mg protein based on Biuret assays). The final concentration for chromogenic substrates was $0.1 \mathrm{mM}$. The formation of $\mathrm{p}$-nitroaniline was monitored at $405 \mathrm{~nm}$ as a function of time.

Amino acid composition and sequence analyses. The amino-acid compositions were determined with a Beckman 6300 amino acid analyzer using a single-column system based on conventional ionexchange chromatography system. The special rapid procedure for the preparation of protein hydrolysates using heat-resistant reusable Pyrex tubes and high temperature $\left(150^{\circ} \mathrm{C}, 1.5 \mathrm{~h}\right)$ for amino acid analysis was essentially according to the previous report (20).

$\mathrm{N}$-Terminal sequence analysis was carried out by automated Edman degradation with a microsequencing sequencer (Model 477A,
Applied Biosystems). The lyophilized column fractions each containing about 1-5 nmoles of protein were dissolved in $200 \mu \mathrm{l}$ of $0.1 \%$ trifluoroacetic acid (TFA) or $0.1 \%$ SDS/0.1\% TFA (1:1 v/v) and $10 \mu \mathrm{l}$ each for sequence determinations.

Determination of cleavage sites using biological peptides as substrates. Cleavage of angiotensin I and bradykinin was measured by incubating $50 \mu$ l of angiotensin I $(1 \mathrm{mg} / \mathrm{ml})$ or bradykinin $(1 \mathrm{mg} / \mathrm{ml}$ ) in $0.05 \mathrm{M} \mathrm{Tris}-\mathrm{HCl}$, pH 7.5, with $5 \mu \mathrm{g}$ of proteases at $37^{\circ} \mathrm{C}$ for $3 \mathrm{~h}$, the mixture was filtered with a microcentrifugation filter (molecular mass cutoff $=10,000$ ). The filtrate was analyzed by HPLC (Bio-Rad Bio-Sil ODS-5S $\mathrm{C}_{18}$ column, $4 \times 250 \mathrm{~mm}$ ). The HPLC was run for 35 min in a linear gradient of $0-75 \%$ solvent B $(95 \%$ acetonitrile containing $0.1 \%$ trifluoroacetic acid (TFA)) with $5 \%$ acetonitrile/0.1\% TFA (solvent A) as the starting and equilibration eluent. The flow rate of column eluates was set at $1 \mathrm{ml} / \mathrm{min}$ and monitored at UV 214 $\mathrm{nm}$. Peak fractions were collected and amino acid compositions were performed as described previously.

Kallikrein-like activity. The kallikrein-like activity was assayed on an SDS-polyacrylamide slab gel (5\% stacking/8\% resolving gel). Small vials containing about $5 \mu \mathrm{g}$ high molecular weight kininogen in $50 \mathrm{mM}$ Tris- $\mathrm{HCl}, \mathrm{pH} 8.0$ buffer were incubated at $37^{\circ} \mathrm{C}$ with $0.2 \mu \mathrm{g}$ of human plasma kallikrein, $0.2 \mu \mathrm{g}$ of TM-VIG or TM-IIG, 0.2 units of Ancrod in a total volume of $10 \mu$ f for various time intervals. After the incubation digestions were stopped by adding $0.1 \%$ SDS $/ 1 \%$ $\beta$-mercaptoethanol and heated at $100^{\circ} \mathrm{C}$ for $5 \mathrm{~min}$. The proteolytic activity was monitored by observing the cleavage patterns of kininogen on Coomassie blue-stained gels after electrophoresis.

Kinin-releasing assay. High molecular-weight kininogen $(20 \mu \mathrm{g})$ was incubated with Tm-VIG or Tm-IIG $(60 \mu \mathrm{g} / \mathrm{ml})$ in $50 \mathrm{mM}$ Tris$\mathrm{HCl}, 1 \mathrm{mM}$ EDTA, pH 8.0 at $37^{\circ} \mathrm{C}$ for $4 \mathrm{~h}$. The released kinin was identified by HPLC (Bio-Rad Bio-Sil ODS-5S $\mathrm{C}_{18}$ column, $4 \times 250$ $\mathrm{mm}$ ) as described previously (21), and its molecular mass analyzed in an LCQ mass spectrometer (Finnigan, San J ose, CA).

Total clottable fibrinogen assay. Fibrinogen assay was calibrated using Fibrinogen Standard (Dade Behring, Marburg, Germany). The Control Plasma $\mathrm{N}$ of normal concentration range (Behring) was used for fibrinogen assay. Various concentrations of venom proteases were incubated with Control Plasma $\mathrm{N}$ for $2 \mathrm{~min}$ at $37^{\circ} \mathrm{C}$, and clotting time measurement was performed with Multifibren $U$ reagent (Behring) on the Humacl ot coagulometer (Human Gesellschaft für Biochemica und Diagnostica mbH, Taunusstein, Germany).

Bleeding-time measurements. Bleeding time of mice was measured by a modification of the method described by Kung et al. (22). Three-month-old ICR mice were used to determine bleeding times. Saline or solutions containing proteases at various concentrations were injected intravenously through a lateral vein of each mouse. After $5 \mathrm{~min}$, the tail was completely transected $2-3 \mathrm{~mm}$ from the tip with a sharp surgical blade. To evaluate bleeding from the incision, a Whatman filter paper was applied to the cutting edge near the clot forming place every $30 \mathrm{~s}$ for $10 \mathrm{~min}$, taking care not to dislodge the clot. Blood that continued to flow from the cut was allowed to fall on the filter paper during 30-s intervals. Bleeding times were determined by measuring the time point with blood stains first disappearing on the filter paper.

In vivo hypotension assay. Blood pressure was assayed by the method as described previously (23). Rats (Sprague-Dawley, body weights of 250-300 g) of either sex were anesthetized with sodium pentobarbital (50-60 mg/kg, intraperitoneally). The trachea was cannulated with a glass cannula for recording respiratory move ments via a volumetric pressure transducer Grass PT 5A (AstroMed. Inc., West Warwick, RI) and also for artificial ventilation with a rodent respirator (Model 680; Harvard Apparatus, Inc., Holliston, $\mathrm{MA}$ ). The right common carotid artery and femoral vein were cannulated with polyethylene tubings filled with heparinized saline. The carotid tubing was attached to a pressure transducer (M odel P23-ID, Statham, Murray Hill, NJ ) connected to a Grass Model 7 polygraph 
(Astro-Med. Inc., West Warwick, RI). The femoral vein was used as an application route for injection of kallikrein-like fibrinogenases or saline.

Statistical analysis. All data are expressed as the mean \pm SEM (n). Student's t test was used to assess the statistical differences.

\section{RESULTS AND DISCUSSION}

Most venoms from snake species induced either bleeding or blood clotting. These activities have been attributed to fibrinogenolytic or fibrinogen clotting enzymes (4-6). In the previous study (17) we have applied multiple-step chromatographies for the isolation and purification of a novel family of kallikrein-like fibrinogenolytic enzymes from the venom of Taiwan habu (Trimeresurus mucrosquamatus, denoted as Tm), named Tm-VIG (with Val-Ile-Gly as the first three $\mathrm{N}$-terminal residues) and Tm-IIG (with Ile-lle-Gly as the first three $\mathrm{N}$-terminal residues), which possess relatively specific and strong activities on $\beta$-chain of human fibrinogen and kallikrein substrate. In this study we have further extended the characterization of these strong proteases with hypotensive effect. The purified native enzymes Tm-VIG and Tm-IIG are acidic proteins with isoelectric points lying between 5.5 to 6.9 and comprise less than $3 \%$ of total crude venom. They can be distinguished from crotalase, thrombin, and kallikrein-like enzymes reported previously from the closely-related crotalid species based on the effects of various protease inhibitors, amino acid compositions and sequence comparison (17).

Fibrinogenolytic activity and substrate specificity of Tm-VIG and Tm-IIG. Both groups of fibrinogenolytic enzymes, i.e., Tm-VIG and Tm-IIG hydrolyzed $\mathrm{B} \beta$ chain of fibrinogen within 5 min with relatively lower activity on $A \alpha$ chain while $\gamma$ chains remained intact even at the end of 120 min (Fig. 1). Tm-VIG and TmIIG can also cleave p-nitroaniline from several synthetic colored peptide substrates. N-benzoyl-Pro-PheArg p-nitroanilide, a specific synthetic substrate for kallikrein-like proteases was most susceptible to hydrolysis by Tm-VIG and Tm-IIG. They also showed relatively high activities towards $\mathrm{N}$-p-tosyl-arginine methyl ester (TAME), indicating that both groups are members of serine proteases family. However D-ValLeu-Lys p-nitroanilide which is a specific substrate for plasmin was demonstrated to be a very poor substrate for these two types of fibrinogenases (unpublished results), attesting to some distinct features of these fibrinogen-digesting proteases as compared with conventional serine proteases involved in the process of blood coagulation.

Incubation of angiotensin I with these two venom proteases resulted in similar degradation patterns. The four major peptide fragments released by specific cleavage on angiotensin I with Tm-VIG and Tm-IIG as determined from amino acid compositions of these pep-

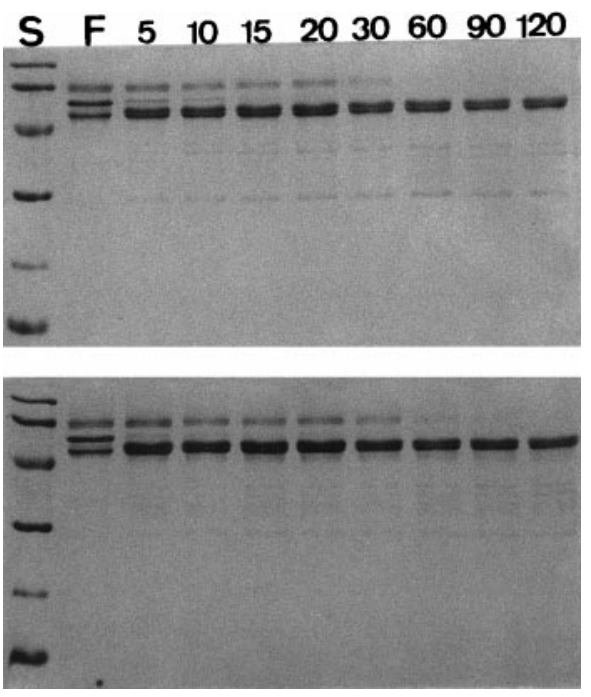

FIG. 1. Time-course study of fibrinogenolytic activity of purified proteases (Tm-VIG and Tm-IIG) on SDS-PAGE. Lane F, purified fibrinogen in the absence of proteases, the three subunit chains are $\mathrm{A} \alpha$, $\mathrm{B} \beta$ and $\gamma$ chains of fibrinogen respectively from the top end downwards; Lane S, standard molecular mass markers (in kDa): phosphorylase b (94), bovine serum albumin (66), ovalbumin (45), carbonic anhydrase (30), soybean trypsin inhibitor (20), and $\alpha$-lactalbumin (14). Lanes with indicated numbers 5-120 denote time-course digestion of fibrinogen with purified proteases Tm-VIG (top) and Tm-IIG (bottom) at $37^{\circ} \mathrm{C}$ for $5,10,15,20,30,60,90$, and $120 \mathrm{~min}$, respectively. Note that the proteases show specific deavages first on $\mathrm{B} \beta$ and then $\mathrm{A} \alpha$ chains with $\gamma$ chain relatively resistant to digestion.

tide fragments are as follows: His-Leu, Asp-Arg-ValTyr, Ile-His-Pro-Phe, and Asp-Arg-Val-Tyr-Ile-HisPro-Phe (corresponding to four major peptide peaks in Fig. 2). This would indicate that these two proteases act on the same sites in angiotensin I. In addition, when kininogen was incubated with purified Tm-VIG or Tm-IIG, the disappearance of kininogen coupled with the formation of the major degradation protein fragment of $58 \mathrm{kDa}$ chain is very similar to that observed for human kallikrein (Fig. 3). The kinin released by Tm-VIG and Tm-IIG from kininogen was further identified by reverse-phase HPLC. Comparison of the fragmentation profiles by mass spectroscopy using synthetic bradykinin as a marker standard identified one of the released peptides as bradykinin (data not shown), pointing to the fact that these two venom proteases may possess genuine hypotensive effect in vivo through bradykinin.

Stability of purified venom proteases with $\beta$-fibrinogenolytic activity. We have carried out thermal stability analysis of Tm-VIG and Tm-IIG by incubating these proteases at different temperatures for $30 \mathrm{~min}$ and examined the fibrinogenolytic activity after heating. It is of surprise to find that these proteases similar to another snake venom protease (ancrod) $(24,25)$ isolated from Malayan pit viper Call oselasma rhodostoma were heat-stable to about $95^{\circ} \mathrm{C}$. They still maintained 


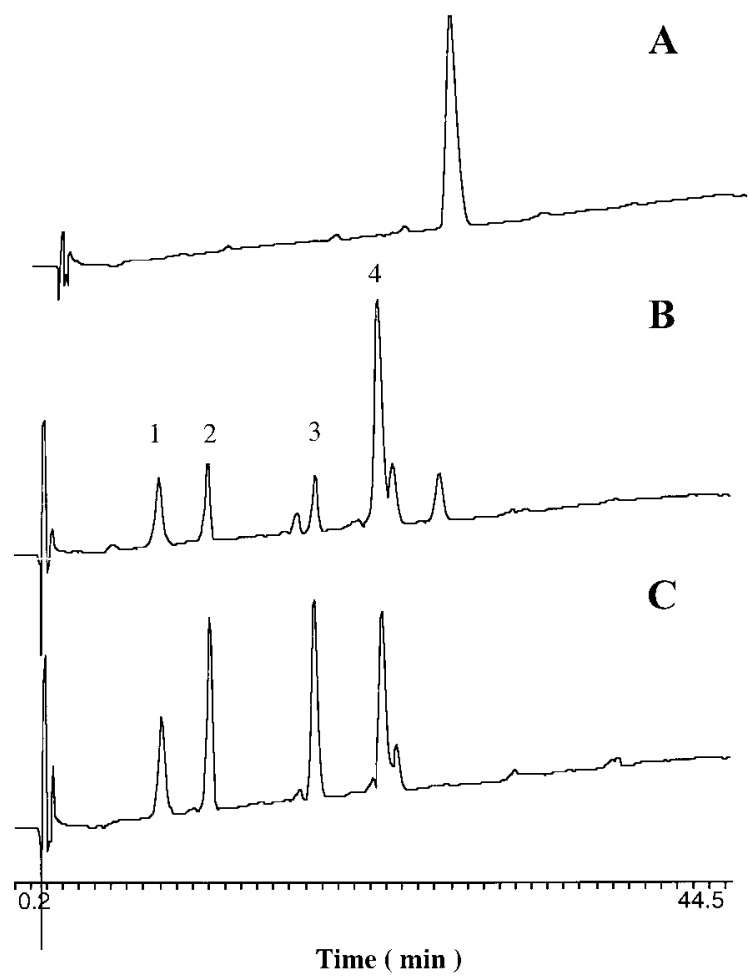

FIG. 2. HPLC chromatograms of angiotensin I cleavage induced by Tm-VIG and Tm-IIG. Chromatography was analyzed by HPLC (Bio-Rad Bio-Sil ODS-5S $\mathrm{C}_{18}$ column, $4 \times 250 \mathrm{~mm}$ ). The HPLC was run for $35 \mathrm{~min}$ in a linear gradient of $0-75 \%$ solvent B $(95 \%$ acetonitrile containing $0.1 \%$ trifluoroacetic acid (TFA)) with $5 \%$ acetonitrile/0.1\% TFA (solvent A) as the starting and equilibration eluent. The flow rate of column eluates was set at $1 \mathrm{ml} / \mathrm{min}$. Each chromatogram represents angiotensin I alone $(A)$ and angiotensin I digested with Tm-VIG (B) or Tm-IIG (C). The labeled peaks indicate 4 major proteolytic fragments by digestion.

their activity at a level of 50-65\% activity even after 30 min heating at this high temperature whereas human thrombin lost activity completely at about $65^{\circ} \mathrm{C}$ (Fig. 4). Both proteases are also more stable than various fibrinogenases previously identified from American rattlesnake venoms $(13,14)$, which are also stable only to about $60-65^{\circ} \mathrm{C}$.

Effects of proteases on total clottable fibrinogen and bleeding time. The conversion of fibrinogen into fibrin plays an important role in coagulation and hemostasis. The final and most defined function of blood coagulation is its effect on plasma clottability. Thus the measurement of clottable fibrinogen in plasma has become a standard protocol for comparison of effects of various biological factors or pharmaceutical agents on the blood clotting process (26). We have used the clottingtime measurement to determine clottable fibrinogen in plasma after treating with Tm proteases. Both venom proteases could prolong clotting times by degrading plasma fibrinogens directly. Total clottable fibrinogen levels were decreased after incubation with purified Tm-VIG/Tm-IIG for 2 min (Fig. 5). However, the blood

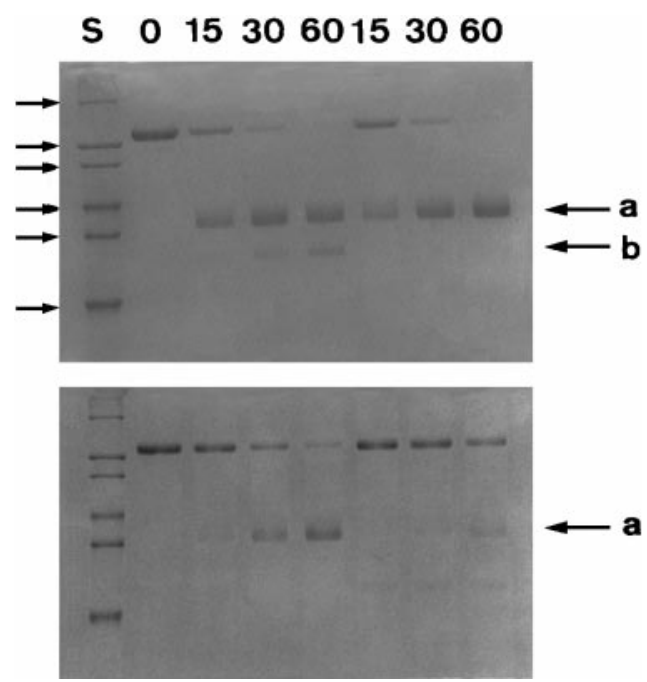

FIG. 3. Time-course study of kallikrein-like activity of venom proteases on SDS-PAGE. High-molecular-weight kininogen (114kDa) was incubated with plasma kallikrein or Tm-VIG (top, left to right), and Tm-IIG or Ancrod (bottom, left to right) at $37^{\circ} \mathrm{C}$ for 15,30 , and $60 \mathrm{~min}$, respectively. Arrows $a$ and $b$ indicate a 58-kDa light-chain fragment and a 45-kDa modified light-chain fragment formed after cleavage with plasma kallikrein. Lane S, standard high-molecular-mass markers (in kDa): myosin (212), $\beta$-galactosidase (116), phosphorylase b (94), bovine serum albumin (66), catalase (57), and aldolase (40).

clotting cannot be induced and clotting-times lengthened indefinitely when the concentrations of proteases were higher than $1 \mu \mathrm{g}$, corroborating the strong fibrin-

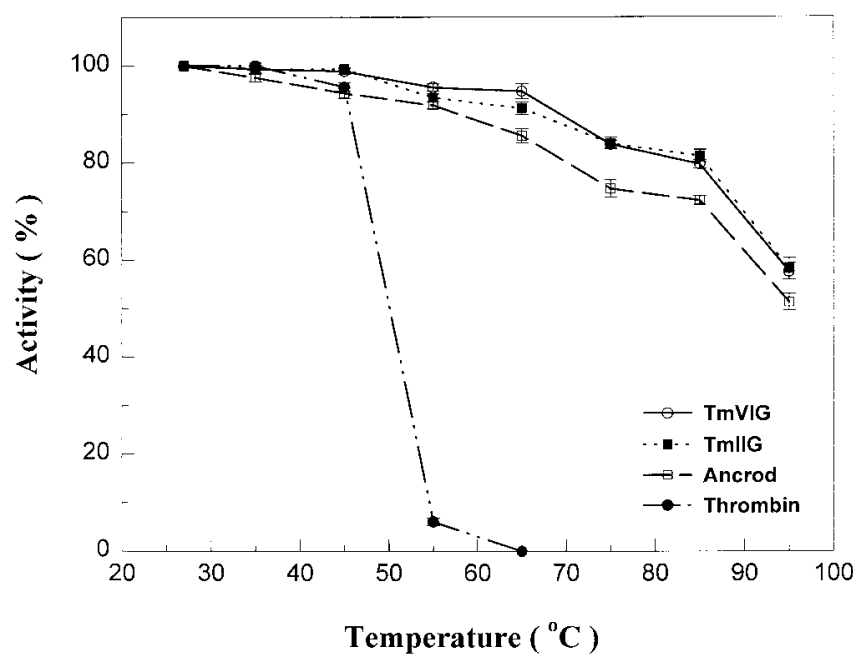

FIG. 4. Effect of temperature on the activity of purified fibrinogenolytic proteases, ancrod and human thrombin. Activity was measured by using $0.1 \mathrm{mM}$ N-benzoyl-Pro-Phe-Arg p-nitroanilide as substrate for Tm-VIG and Tm-IIG, and $0.1 \mathrm{mM} \mathrm{N-p-tosyl-Gly-Pro-Arg}$ p-nitroanilide for ancrod and thrombin due to different substrate specificities among these proteases. Percent activity at different temperatures with reference to that at the ambient room temperature (100\%) was compared for these four proteases. The proteolytic activities using synthetic chromogenic substrates were measured on a spectrophotometer at $405 \mathrm{~nm}$. Data are presented as mean \pm SEM $(n=3)$. 


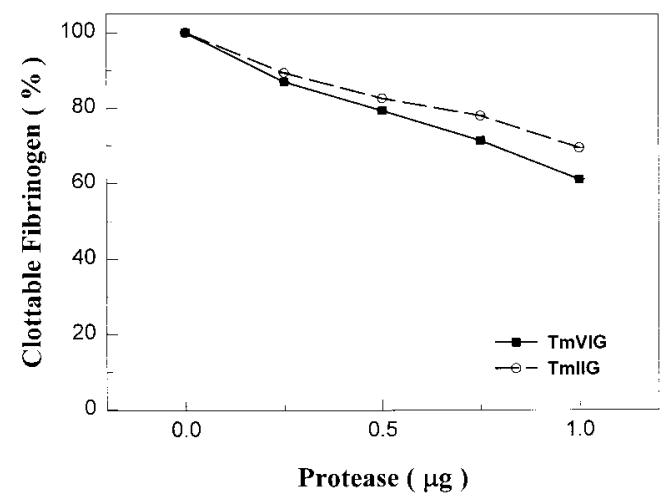

FIG. 5. Effect of kallikrein-like Tm proteases on apparent fibrinogen concentration in plasma measured with a coagulometer. The samples from human Control Plasma $\mathrm{N}$ were mixed with various concentrations of venom proteases. Values are given as percent of original fibrinogen concentration measured in the absence or presence of venom proteases using the Control Plasma N solution without protease as reference. Data are assayed in triplicate measurements.

ogenolytic activity associated with these two novel venom proteases. The bleeding times measured after surgical transections on tails upon intravenous administration of proteases to mice were significantly prolonged in a dose-dependent manner (Fig. 6). It is noteworthy that the anti-clotting or bleeding effect of TmVIG was stronger than that of Tm-IIG significantly, which deserves a further study on detailed structural determination of these two proteases in the future. Similar to clottable fibrinogen assays, the bleeding times were found to lengthen to more than $10 \mathrm{~min}$ and hemorrhagic side-effects appeared when amounts of proteases injection were higher than $5 \mu \mathrm{g}$ per mouse. Therefore from the in vitro clottable fibrinogen assays and in vivo bleeding time measurements, it is conceivable that Tm-VIG and Tm-IIG may be directly involved in decreasing the levels of fibrinogen in the plasma through defibrinogenation. In contrast, ancrod and batroxobin, which are members of thrombin-like enzymes, can deave specifically fibrinopeptides A or B from fibrinogen resulting in formation of fibrin dots (27).

Hypotensive effects of Tm proteases on experimental rats. The hypotensive effects of proteases on rat blood pressure were investigated by injecting these proteins into cannulated rats. A significant blood pressure drop was observed with Tm-VIG injection (Fig. 7). Injection of Tm-IIG showed a milder effect. The hypotensive effect exhibited by these venom proteases are likely due to their inherent kallikrein-like activity. There is a possibility that Tm-VIG and Tm-IIG may directly affect the blood coagulation pathway by specifically cleaving $B \beta$ chains of animal fibrinogens and act like plasma kallikreins to cleave kininogen. This kallikrein-like activity is especially intriguing since the reported $\alpha$-fibrinogenases like ancrod did not show such a high specificity against kininogen (data not shown). Moreover both Tm-VIG and Tm-IIG demonstrated no obvious ability to induce or inhibit platelet aggregation, in great contrast with some venom antithrombotic factors reported in the literature.

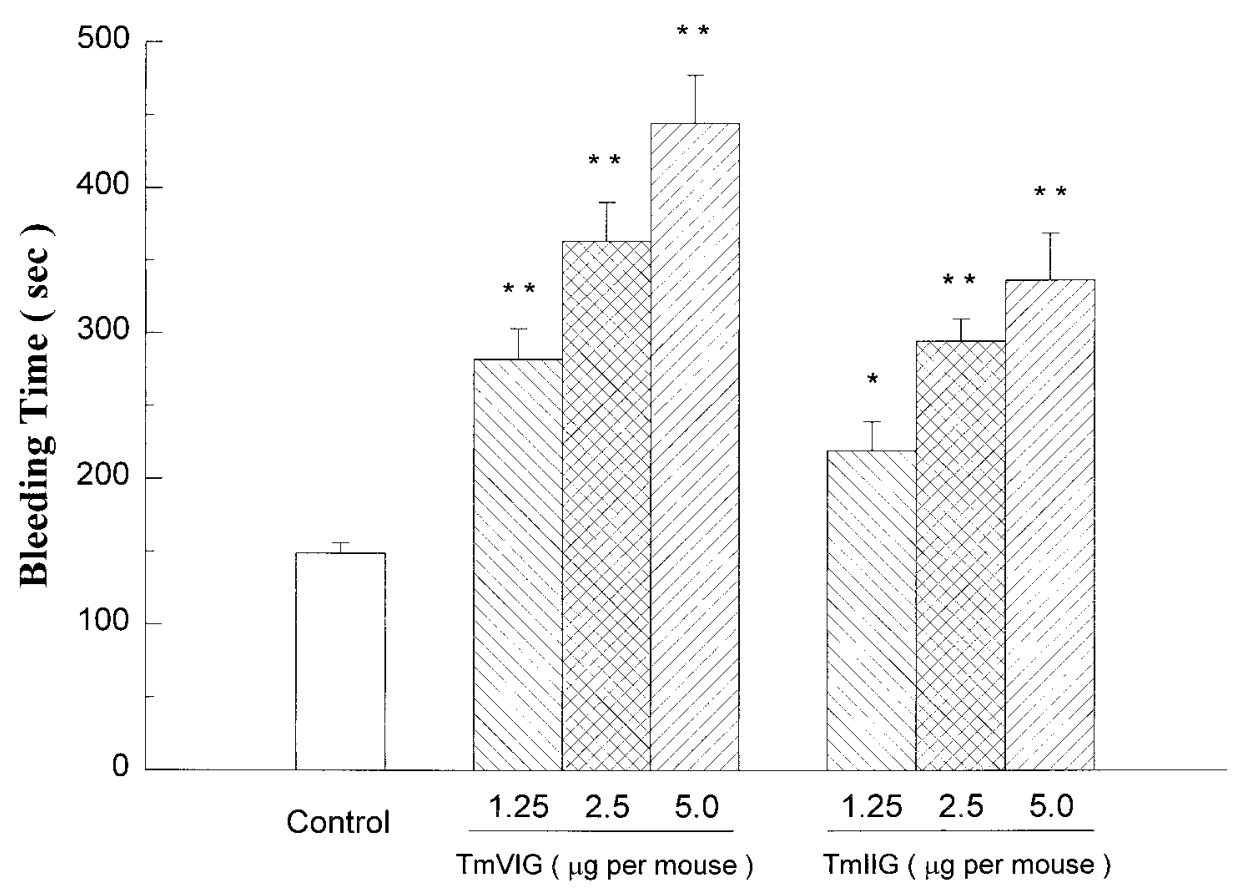

FIG. 6. Effect of kallikrein-like Tm proteases on tail bleeding time in mice by a filter paper method. Bleeding time was measured 5 min after the intravenous administration of saline or various doses of proteases. Data are presented as mean \pm SEM ( $n=6$ ), with significance levels at $* \mathrm{P}<0.05$ or $* * \mathrm{P}<0.01$ as compared with the control. 


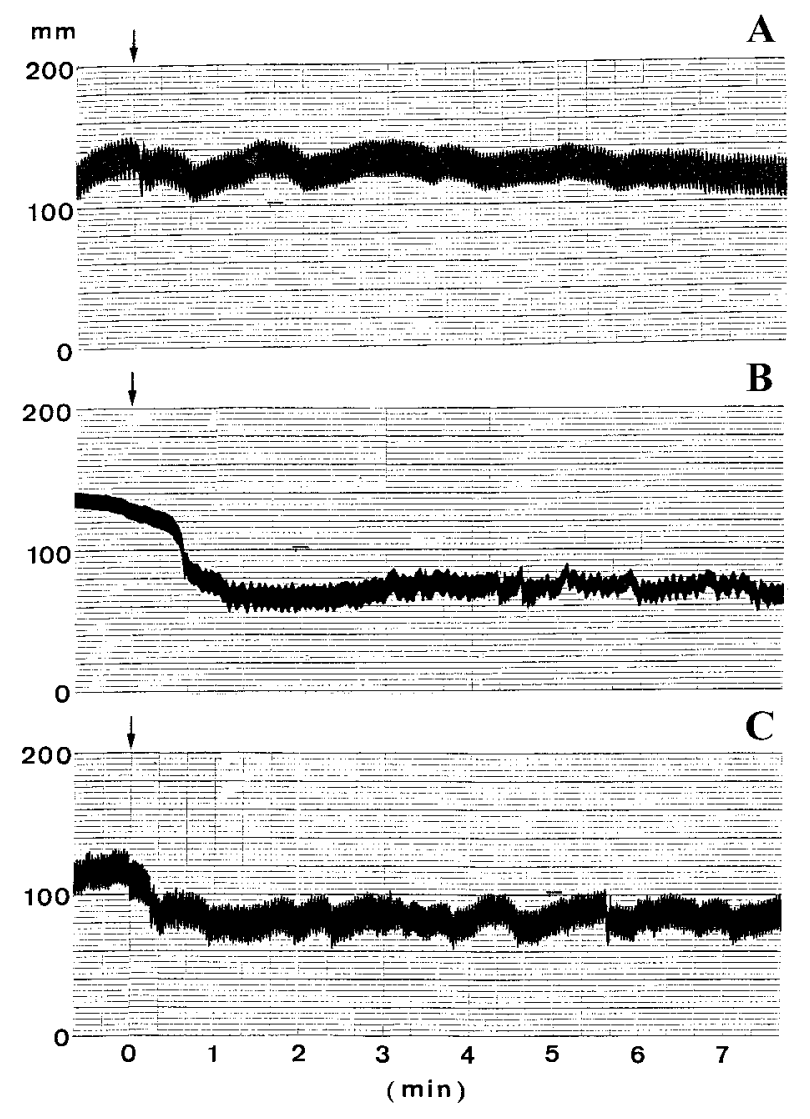

FIG. 7. Hypotensive effect on rat blood pressure of kallikreinlikeTm fibrinogenases from Taiwan habu. (A) Blood pressure change after intravenous (i.v.) injection with normal saline; (B) blood pressure change after i.v. injection of Tm-VIG (0.5 $\mu \mathrm{g} / \mathrm{g}$ weight); (C) blood pressure change after i.v. injection of Tm-IIG (0.5 $\mu \mathrm{g} / \mathrm{g}$ weight). Arrows indicate starting points for sample injections. Note that Tm-IIG seemed to show a lower hypotensive activity than Tm-VIG under similar experimental conditions.

In 1949 Rocha e Silva et al. (28) first reported that trypsin and certain snake venoms acted on plasma globulin to produce a substance that lowered blood pressure. They termed this substance bradykinin. It is likely that kallikrein-like $\beta$-fibrinogenases reported here may be the snake venom enzymes responsible for the generation of bradykinin from endogenous HMW kininogen to lower blood pressure. The in vivo hypotensive effect on rats by these enzymes (Fig. 7) attested to the effectiveness of using these fibrinogenases as antihypertensive agents. An additional activity of these Tm fibrinogenases, the degradation of the hypertensive peptide angiotensin I (Fig. 2), may also potentiate hypotensive effect exhibited by these Tm venom proteases. Further functional and structural characterization regarding the structure/biological activity correlation should shed some insight into the mechanism underlying their kallikrein-like hypotensive effect and fibrinogenolytic action.

In conclusion, enzymes which interfere with haemostasis in vertebrates are common components in snake venoms. Two reptilian venom proteases ancrod $(24,25)$ and batroxobin $(29,30)$ from viperid snakes have been found clinically useful for the treatment of thrombotic diseases $(31,32)$ during the past three decades. Venom proteolytic enzymes with kallikrein-like activity characterized in this study are strong and very heat-stable fibrinogenolytic proteases first reported from Taiwan habu, which is an evolutionarily more remote crotalid species when compared with two viperid snake species that contain batroxobin and ancrod. The comparison of activity and thermal stability of habu proteases with ancrod and batroxobin indeed shows great potentials in exploiting these novel $\beta$-fibrinogenases with kallikrein-like activity as effective antithrombotic and antihypertensive agents.

\section{ACKNOWLEDGMENTS}

This work was supported in part by Academia and the National Science Council (NSC Grants 87-2311-B-002-068, 88-2311-B-002061 and 89-2311-B-001-190 to S.-H. Chiou), Taipei, Taiwan. This report will be submitted as part of a dissertation by C.-C. Hung to National Taiwan University in partial fulfillment of the degree of Doctor of Philosophy. We thank Professor Wan-Wan Lin at the Department of Pharmacology, College of Medicine, National Taiwan University for assisting hypotensive assays for the isolated fibrinogenases.

\section{REFERENCES}

1. Ouyang, C. (1957) J . Formosan Med. Assoc. 56, 435- 448.

2. Meaume, J . (1966) Toxicon 4, 25-58.

3. Kini, R. M., and Evans, H. J . (1990) Toxicon 28, 1387-1422.

4. Brinkhous, K. M., and Smith, S. V. (1988) in Hematology, Haemostasis and Animal Venoms (Pirkle, H., and Markland, F. S., J r., Eds.), Vol. 7, pp. 363-375, Marcel Dekker, New York.

5. Stocker, K. F. (1990) in Medical Use of Snake Venom Proteins (Stocker, K. F., Ed.), pp. 97-160, CRC Press, Boston, MA.

6. Tu, A. T. (1982) in Rattlesnake Venoms: Their Actions and Treatment (Tu, A. T., Ed.), pp. 247-312, Marcel Dekker, New York.

7. Serrano, S. M. T., Mentele, R., Sampaio, C. A. M., and Fink, E. (1995) Biochemistry 34, 7186-7193.

8. Markland, F. S., J r. (1998) Thromb. Haemostasis 79, 668674.

9. Markland, F. S., and Damus, P. S. (1971) J . Biol. Chem. 246, 6460- 6473.

10. Bjarnason, J. B., and Tu, A. T. (1978) Biochemistry 17, 33953404.

11. Pandya, B. V., and Budzynski, A. Z. (1984) Biochemistry 23, 460- 470 .

12. Bjarnason, J . B., Barish, A., Direnzo, G. S., Campbell, R., and Fox, J. W. (1983) J . Biol. Chem. 258, 12566-12573.

13. Chiou, S.-H., Hung, C.-C., and Lin, C.-W. (1992) Biochem. Int. 26, 105-112.

14. Chiou, S.-H., Hung, C.-C., and Huang, K.-F. (1992) Biochem. Biophys. Res. Commun. 187, 389-396.

15. Ouyang, C., and Teng, C. M. (1976) Biochim. Biophys. Acta 420, 298-308. 
16. Huang, K.-F., Hung, C.-C., and Chiou, S.-H. (1993) Biochem. Mol. Biol. Int. 31, 1041-1050.

17. Hung, C.-C., Huang, K.-F., and Chiou, S.-H. (1994) Biochem. Biophys. Res. Commun. 205, 1707-1715.

18. Laemmli, U. K. (1970) Nature 227, 680- 685.

19. Roe, J . H. (1955) J . Biol. Chem. 212, 335-343.

20. Chiou, S.-H. (1988) Biochem. Int. 17, 981-987.

21. Fiedler, F., and Geiger, R. (1988) Methods Enzymol. 163, 257262.

22. Kung, S. H., Hagstrom, J. N., Cass, D., Tai, S. J ., Lin, H. F., Stafford, D. W., and High, K. A. (1998) Blood 91, 784-790.

23. Lin, W. W., Chen, Y. M., Lee, S. Y., and Lee, C. Y. (1991) Asia-Pacific J . Pharmacol. 6, 277-286.

24. Burkhart, W., Smith, G. F. H., Su, J . L., Parikh, I., and LeVine, H. III. (1992) FEBS Lett. 297, 297-301.
25. Au, L. C., Lin, S. B., Chou, J . S., Teh, G. W., Chang, K. J ., and Shih, C. M. (1993) Biochem. J . 294, 387-390.

26. Gaffney, P. J ., and Wong, M. Y. (1992) Thromb. Haemostasis 68, 428- 432.

27. Markland, (1998) Toxicon 36, 1749-1780.

28. Rocha e Silva, M., Beraldo, W. T., and Rosenfeld, G. (1949) Am. J . Physiol. 156, 261-273.

29. Holleman, W. H., and Weiss, L. J . (1976) J . Biol. Chem. 251, 1663-1669.

30. Itoh, N., Tanaka, N., Mihashi, S., and Yamashina, I. (1987) J . Biol. Chem. 262, 3132-3135.

31. Stocker, K. (1978) in Handbook of Experimental Pharmacology (Markwardt, F., Ed.), Vol. 46, pp. 451-484, Springer-Verlag, Berlin.

32. Latollo, Z. S. (1978) Thromb. Haemostasis 39, 768-774. 